\title{
Enhancement of anti-tumor immunity by high levels of Th1 and Th17 with a combination of dendritic cell fusion hybrids and regulatory $T$ cell depletion in pancreatic cancer
}

\author{
MASASHI YAMAMOTO ${ }^{1}$, TAKASHI KAMIGAKI ${ }^{1}$, KIMIHIRO YAMASHITA ${ }^{1}$, \\ YUICHI HORI ${ }^{2}$, HIROSHI HASEGAWA ${ }^{1}$, DAISUKE KURODA ${ }^{1}$, HIROAKI MORIYAMA ${ }^{3}$, \\ MASAO NAGATA ${ }^{3}$, YONSON KU ${ }^{2}$ and YOSHIKAZU KURODA ${ }^{1}$ \\ Department of Surgery, Divisions of ${ }^{1}$ Gastro-intestinal Surgery and ${ }^{2}$ Hepato-Biliary-Pancreatic Surgery, \\ ${ }^{3}$ Department of Internal Medicine, Division of Internal and Geriatric Medicine, Kobe University \\ Graduate School of Medicine, Kusunoki-cho, Kobe, Hyogo 650-0017, Japan
}

Received March 5, 2009; Accepted April 30, 2009

DOI: 10.3892/or_00000442

\begin{abstract}
Regulatory T cells (Tregs) play an important role in immunological self-tolerance and protect the host from autoimmune disease. However, in cancer immunity, Tregs might block anti-tumor immune responses. Therefore, the depletion of Tregs using a specific agent that suppresses its function or population, such as an anti-CD25 antibody, could promote anti-tumor immune responses. In the present study, a cytotoxicity assay, enzyme-linked immunosorbent spot (ELISPOT) assay and measuring cytokine secretion, were used to study the efficacy of Treg depletion by anti-CD25 antibody added to a dendritic cell/tumor cell (DC/TC) fusion hybrid vaccine in a murine pancreatic cancer model. All the mice treated with the combined therapy of fusion hybrid vaccine and Treg depletion rejected tumor growth in a challenging test, although the rejection rate was $20 \%$ both for mice that received the fusion hybrids alone or Treg depletion alone. In addition, combined therapy showed a significantly improved survival in comparison to other treatment or control groups. The NK cell activity for DC/TC fusion + Treg depletion was significantly higher than that for the other treatment groups. Cytotoxic T lymphocyte (CTL) activity for DC/TC could potentially be enhanced by the addition of Treg depletion therapy. The treatments including DC/TC fusion induced IFN- $\gamma$ secreting effector cells in ELISPOT assays. Furthermore, a cytometric beads array assay used to measure cytokine secretion showed that DC/TC fusion + Treg depletion stimulated the highest
\end{abstract}

Correspondence to: Dr Takashi Kamigaki, Department of Surgery, Division of Gastro-intestinal Surgery, Kobe University Graduate School of Medicine, Kusuoki-cho 7-5-2, Chuoku, Kobe, Hyogo 650-0017, Japan

E-mail: tkamy@med.kobe-u.ac.jp

Key words: dendritic cell/tumor cell fusion hybrids, regulatory T cell, Th1/Th2 cytokines, interleukin-17, pancreatic cancer levels of IFN- $\gamma$ Th1/Th2 ratios and Th17. These results demonstrate that Treg depletion combined with DC/TC fusion hybrid vaccine enhanced the efficacy of immunotherapy in pancreatic cancer by activating CTLs and NK cells.

\section{Introduction}

Dendritic cells (DCs) are potent antigen-presenting cells (APCs) which are essential to control the defense mechanism of the immune system against tissue-specific antigens, such as viral proteins or auto-antigens. DCs also play an important role in the induction of anti-tumor immune responses by stimulating the generation and proliferation of tumor-specific cytotoxic $\mathrm{T}$ lymphocytes (CTLs) and helper T cells $(1,2)$. A large number of immunotherapeutic strategies have used DC vaccines loaded with tumor antigen associated peptides, DNA or RNA. DC vaccines pulsed with tumor lysates or stressed tumor cells have also been shown to elicit an anti-tumor effect in animals and clinical trials (3-5).

The vaccination with DC and tumor cell fusion hybrids (DC/TC hybrids) shows promising efficacy in murine tumor models (6-8). DC/TC hybrids can express multiple tumor antigens in the context of MHC class-I and/or class-II molecules and induce polyclonal immune responses against tumor antigens. Previously, vaccination of DC/TC hybrids was effective for the induction of anti-tumor activity in humans (9-12). However, in cancer immunotherapy, the efficacy of vaccinations is often insufficient to activate the anti-tumor immune response, because of the immune-suppressive effect induced by inhibitory cells, such as regulatory T cells (Treg) in the tumor microenvironment. Treg are defined by their expression of $\mathrm{CD} 4$, the interleukin-2 receptor component $\mathrm{CD} 25$ and intracellular expression of the transcription factor FoxP3 and play an important role in immunological selftolerance and in suppressing excessive immune responses (13-16). Tregs suppress the activities or function of CD4(+), CD8(+) T cells, natural killer (NK) cells, and NKT cells through several pathways, such as the secretion of suppressive cytokines $e . g$. TGF- $\beta$ and IL-10, cell-to-cell contacting 
mechanism, and the modulation of APCs function (17-19). In animal models, murine pancreatic adenocarcinoma Pan02 tumors promoted Tregs through TGF- $\beta$ secretion (20). To overcome the immune suppression of Tregs, the depletion of Tregs could therefore promote anti-tumor immune responses in human (21).

The present study compared the anti-tumor efficacy of the following combination of DC-based vaccine and Treg depletion: Treg depletion with an anti-CD25 antibody, vaccination with DC/TC fusion hybrids and a combination of both in Pan02 tumor models. The relative degree of anti-tumor protection in vivo and the efficacy of anti-tumor effector cells were evaluated by comparing the results of a cytotoxicity assay, while measuring the cytokine secretion from effector cells and enzyme-linked immunosorbent spot assays (ELISPOT).

\section{Materials and methods}

Mice. Female C57BL/6 mice aged 6-8 weeks purchased from CLEA Japan Inc (Tokyo, Japan). The mice were housed under pathogen-free conditions at the Animal Maintenance Facility at Kobe University School of Medicine and treated according to the university ethics guidelines for animal use in research.

Pancreatic cancer cell lines. The murine pancreas adenocarcinoma cell line Pan02 was kindly provided by Dr David C. Linehan (Washington University School of Medicine, St. Louis, MO). It was cultured in RPMI-1640 medium (Sigma-Aldrich Inc., St. Louis, MO) supplemented with $10 \%$ fetal bovine serum, $100 \mu \mathrm{g} / \mathrm{ml}$ streptomycin and $100 \mathrm{U} / \mathrm{ml}$ penicillin at $37^{\circ} \mathrm{C}$ in a humidified atmosphere of $5 \%$ $\mathrm{CO}_{2}$.

Dendritic cells. Bone marrow-derived DCs were generated, as previously described (22). Briefly, bone marrow harvested from femurs and tibiae of C57BL/6 mice, was filtered through $100 \mu \mathrm{m}$ nylon cell strainer (BD Biosciences-Labware, San Jose, CA). The cells were aliquoted to 24 -well plates (BD Biosciences-Labware) at $1 \times 10^{6}$ cells $/ \mathrm{ml}$ RPMI-1640 supplemented with $10 \mathrm{ng} / \mathrm{ml}$ recombinant murine GM-CSF (Peprotech Inc., Rocky Hill, NJ, USA), $20 \mathrm{ng} / \mathrm{ml}$ recombinant murine IL-4 (Peprotech Inc.) and $50 \mu \mathrm{M}$ 2-mercaptoethanol (2-ME). After 7 days of culture, the non-adherent and loosely adherent cells were harvested, washed and then used for experiments.

Generation of DCs/tumor cell fusion hybrids. Bone marrow derived DCs were fused to irradiated Pan02 cells ( $30 \mathrm{~Gy}$ ) at a ratio of $2: 1$, respectively, in a solution of polyethylene glycol in DMSO (Sigma-Aldrich), as described previously (23). Briefly, the DC/tumor cell suspension was washed twice with conditioned medium and was then resuspended in $1 \mathrm{ml}$ of $50 \%$ polyethylene glycol added over $1 \mathrm{~min}$ and stirred gently for 1-2 min. Pre-warmed RPMI-1640 was then slowly added by gently stirring the suspension. After 2 min at $37^{\circ} \mathrm{C}$, the resultant mixture of the cells was grown overnight in a culture medium containing GM-CSF. In the present study, loosely adherent, DC/irradiated tumor cell fusion hybrids were used for vaccination at $24 \mathrm{~h}$ after chemical fusion.

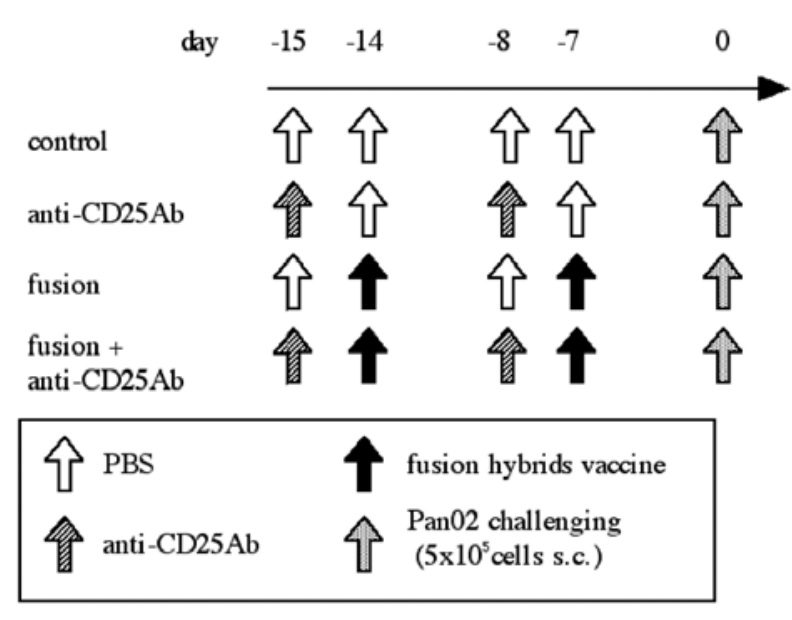

Figure 1. Treatment schedule for various combinations of anti-CD25Ab and vaccine. Twenty C57BL/6 mice were divided into four immunization groups: PBS control; anti-CD25Ab alone; fusion alone; fusion + anti-CD25Ab. The injection of $100 \mu \mathrm{g}$ anti-CD25Ab was given twice in the peritoneum on days 15,8 in the groups of anti-CD25Ab monotherapy or fusion + anti$\mathrm{CD} 25 \mathrm{Ab}$. Fusion hybrids vaccine was injected twice in the left inguinal region with $1 \times 10^{6} \mathrm{DC} / \mathrm{TC}$ fusion hybrids cells on day 14,7 in groups of fusion including regimens. The mice were injected subcutaneously in the right flank region with $5 \times 10^{5}$ Pan02 cells on day 0 .

Treg depletion and vaccination of fusion hybrids. C57BL/6 mice were divided into four groups. Each group was injected subcutaneously in the right inguinal region with $5 \times 10^{5} \mathrm{Pan} 02$ cells on day 0 . There were four immunization groups: group 1 , no anti-CD25Ab and no fusion (PBS control); group 2, anti-CD25Ab; group 3, fusion; group 4, anti-CD25Ab and fusion. Treg depletion by anti-CD25Ab was accomplished by 2 intraperitoneal injections of $100 \mu \mathrm{g}$ of anti-mouse CD25 antibody (PC61.5, functional grade purified anti-mouse CD25 antibody, eBioscience Inc. San Diego, CA) on days 15 and 8. Vaccinations of $1 \times 10^{6} \mathrm{DC} / \mathrm{TC}$ fusion hybrids were injected subcutaneously in the left inguinal region twice a day after antibody injection (days 14 and 7; Fig. 1).

Tumor measurement. The long axis and the short axis of the tumor were measured subcutaneously twice a week until 60 days after a subcutaneous tumor challenge. The tumor volume was calculated by (the long axis) $\mathrm{x}$ (the short axis) ${ }^{2}$ $\mathrm{x} 1 / 2\left(\mathrm{~mm}^{3}\right)$.

Cytotoxicity assay. C57BL/6 mice were divided into the previous four groups and each group contained three mice. Immunizations were done, as described above. At 7 days after tumor challenge, splenocytes were isolated and stimulated with $1.0 \times 10^{5}$ Pan02 cells irradiated with 30 Gy for a day in RPMI-1640 supplemented with $10 \%$ FBS, $100 \mu \mathrm{g} / \mathrm{ml}$ streptomycin and $100 \mathrm{U} / \mathrm{ml}$ penicillin, $50 \mu \mathrm{M}$ 2-ME. Splenocytes were collected and tested for their cytotoxic ability. Effector (E) cells were added to target $(\mathrm{T})$ cells (Pan02 for CTL assay, YAC-1 for NK cell assay) labeled with ${ }^{51} \mathrm{Cr}$ and cultured for $4 \mathrm{~h}$ at various $\mathrm{E} / \mathrm{T}$ ratios. The percent cytotoxicity was calculated from the release of ${ }^{51} \mathrm{Cr}$ as follows: [(experimental release)-(target spontaneously release)]/ [(target maximum release) - (target spontaneously release)] x 100 . 


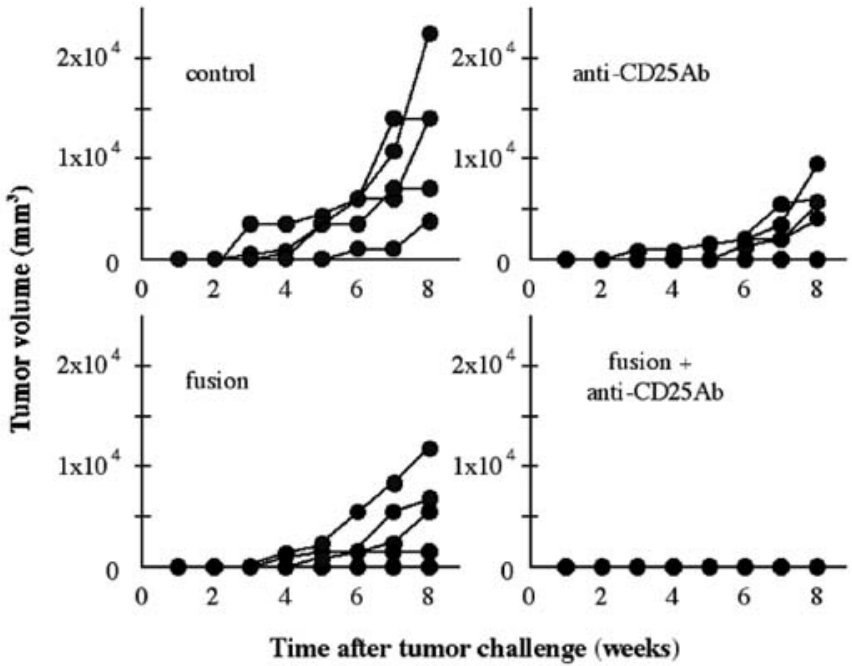

Figure 2. The tumor volume after Pan02 challenge for various treatment groups. The experimental schedule is as described in Fig. 1. The tumor was measured along the long axis and the short axis subcutaneously until 60 days after the tumor challenge. The tumor volume was calculated by (the long axis) $\mathrm{x}$ (the short axis) $)^{2} \times 1 / 2\left(\mathrm{~mm}^{3}\right)$. No mice that received the combined fusion + anti-CD25Ab therapy revealed any tumor growth.

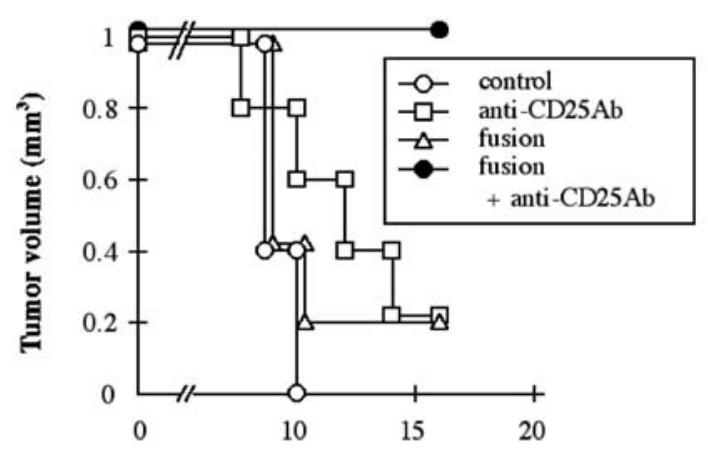

Time after tumor challenge (weeks)

Figure 3. Survival rate of each treatment group. The figure dipicts KaplanMeier curves. No mouse died within 16 weeks after tumor challenge in the fusion + anti-CD25Ab group. The survival rate of the fusion + anti-CD25Ab group was significantly longer than that of other groups based on the log-rank test (vs. PBS control: $\mathrm{p}=0.016$, vs. anti-CD25Ab: $\mathrm{p}=0.029$, vs. fusion: $\mathrm{p}=0.025)$.

Cytokine assay. The mice were divided into four groups as previously described and each group contained three mice. Immunizations and stimulation for a day were done same as described above. The splenocytes were collected and assessed by murine IFN- $\gamma$ ELISPOT (BD Bioscience). The supernatant was collected and secretion of various cytokines was measured by a cytometric bead array assay (CBA Mouse Inflammation kit, BD Pharmingen) or mouse Interleukin (IL)-17 enzymelinked immunosorbent assay (ELISA, Bender Med System Gmbh, Vienna, Austria). In the ELISPOT assay, $1 \times 10^{6}$ splenocytes per well were cultured with $1 \times 10^{3} \mathrm{Pan} 02$ cells at $37^{\circ} \mathrm{C}$ in a humidified atmosphere of $5 \% \mathrm{CO}_{2}$ in precoated wells. After $18 \mathrm{~h}$ of co-culture, the plate was developed according to manufacturer's protocol and the spot forming cells (SPC) were counted by using an ELISPOT reader and analyzed with ELISPOT software (Cellular Technology Ltd. Shaker
Heights, OH). In the CBA assays, 6 cytokines, IFN- $\gamma$, IL-6, IL-10, IL-12p70, TNF- $\alpha$ and MCP-1 were measured in the supernatant of the effector cells stimulated with Pan02 cells. In IL-17 ELISA, mouse IL-17 was also measured in the supernatant of splenocyte after 1 or 5 days culture with Pan02 cells.

Statistical analysis. Survival data were described as KaplanMeier curves. Statistical significance was determined using Student's t-test and one-factor ANOVA. P-values $<0.05$ were considered to be significant.

\section{Results}

Treg depletion and fusion vaccination suppressed tumor growth and improved survival. Twenty C57BL/6 mice were divided into four groups and received a combination of Treg depletion and fusion vaccination. All mice were injected with $5 \times 10^{5}$ Pan02 cells subcutaneously on day 0 and were observed for tumor growth. The tumor size of each mouse was measured until day 60 and the survival was followed up until 120 days after tumor inoculation. All mice immunized both with fusion and anti-CD25Ab rejected tumor growth (5/5). Mice immunized with fusion or anti-CD25Ab demonstrated $20 \%$ rejection rate (1/5; Fig. 2). At 56 days after tumor injection, the mean tumor volume for PBS control, anti-CD25Ab, and fusion were $12,050 \pm 6,616 \mathrm{~mm}^{3}, 6,094 \pm 2,335 \mathrm{~mm}^{3}$ and $6,813 \pm 2,996 \mathrm{~mm}^{3}$, respectively. There are no notable differences in the tumor size between mice given the anti$\mathrm{CD} 25 \mathrm{Ab}$ and fusion hybrid vaccine, however $\sim 50 \%$ tumor growth inhibition was observed in mice that received anti$\mathrm{CD} 25 \mathrm{Ab}$ or fusion in comparison to the control. The median survival time was: PBS control, 59 days (range, 56-63); antiCD25Ab, 71 days (range, 56-112); and fusion, 69 days (range, 56-112); however, for the fusion + anti-CD25Ab group, all mice survived 120 days post-inoculation of tumor cells (Fig. 3). For the control group, all mice died within 10 weeks after inoculation of Pan02 cancer cells. The combination of Treg depletion and fusion vaccination showed a significantly longer survival than Treg depletion only or fusion vaccination only $(\mathrm{p}<0.03)$.

CTL and NK cell activity enhanced by combination therapy of Treg depletion and fusion vaccination. The Pan02-specific CTL activity and YAC-1-specific NK cell activity were examined using a $4 \mathrm{~h}{ }^{51} \mathrm{Cr}$ releasing cytotoxity assay. CTL activity for the fusion + anti-CD25Ab group was 10.2, 13.5 and $14.2 \%$ at the $\mathrm{E} / \mathrm{T}$ ratio $12.5: 1,25: 1$ and 50:1, respectively (Fig. 4). For combined therapy, the CTL activity was significantly higher than that for the control at an E/T ratio of 50:1 (15.8 vs. 9.6\%, p<0.05). Although, the CTL activity for fusion + anti-CD25Ab was found to be highest at each E/T ratio, no significant difference was seen between three therapy groups for Pan02-specific CTL activity at the 50:1 E/T ratio. The NK cell activity of fusion + anti-CD25Ab group at the E/T ratio $12.5: 1,25: 1$ and $50: 1$ was $22.7,50.1$ and $61.3 \%$, respectively (Fig. 4). The NK cell activity for fusion only or fusion + anti-CD25Ab group was significantly higher than that for PBS control $(\mathrm{p}<0.005)$. Fusion + anti-CD25Ab combined therapy showed a significantly higher level of the 
Table I. Cytokine production by splenocytes from mice immunized with fusion hybrid vaccine and Treg depletion.

\begin{tabular}{lccccc}
\hline Immunization group & IFN- $\gamma(\mathrm{pg} / \mathrm{ml})$ & IL-6 $(\mathrm{pg} / \mathrm{ml})$ & IL-10 $(\mathrm{pg} / \mathrm{ml})$ & IFN- $/ \mathrm{IL}-6$ & IFN- $\gamma / \mathrm{IL}-10$ \\
\hline Control & $3.8 \pm 0.2$ & $82.4 \pm 6.2$ & N.D & $0.046 \pm 0.0012$ & - \\
Anti-CD25Ab & $17.9 \pm 10.2$ & $113.2 \pm 29.1$ & $9.8 \pm 6.7$ & $0.18 \pm 0.13$ & $3.1 \pm 1.2$ \\
Fusion & $210.9 \pm 18.1^{\mathrm{a}}$ & $942.6 \pm 155.8$ & $115.7 \pm 26.2$ & $0.23 \pm 0.044$ & $1.9 \pm 0.4$ \\
Fusion + anti-CD25Ab & $594.9 \pm 88.7^{\mathrm{a}}$ & $1387.2 \pm 174.1$ & $152.2 \pm 60.8$ & $0.43 \pm 0.018$ & $4.3 \pm 1.4^{\mathrm{b}}$ \\
\hline
\end{tabular}

Splenocytes from mice immunized with PBS, fusion hybrid vaccination, Treg depletion by anti-CD25Ab and fusion hybrid vaccination + Treg depletion by anti-CD25Ab were co-cultured with irradiated Pan02 cells. The supernatant of each culture was collected and examined by CBA

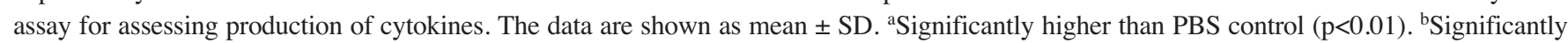
higher than fusion hybrids $(\mathrm{p}=0.045)$.

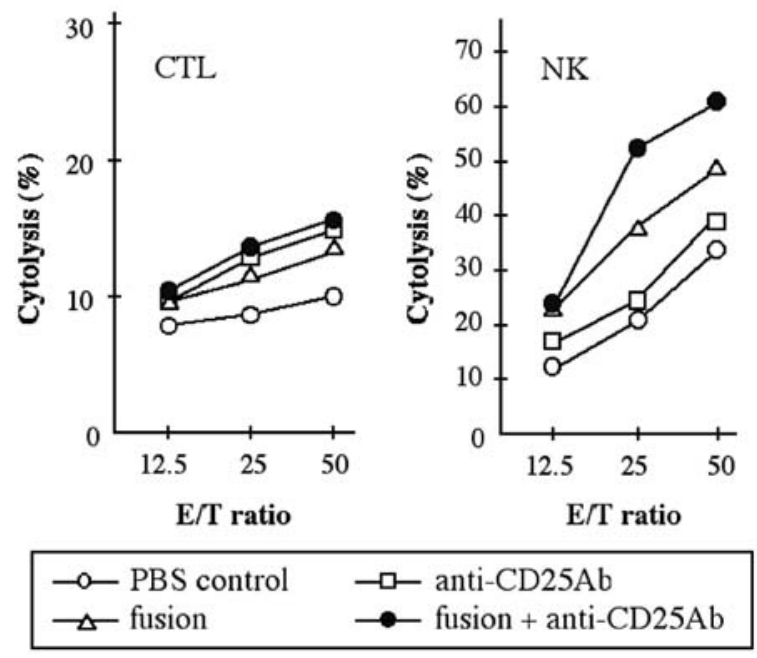

Figure 4. C57BL/6 female mice were divided into four groups: PBS control, anti-CD25Ab alone, fusion alone and anti-CD25Ab + fusion. Seven days after tumor challenge, splenocytes were isolated and were co-cultured with $1.0 \times 10^{5}$ irradiated Pan02 cells for one day. Splenocytes were collected and tested for their cytotoxic ability as effector cells. Effector $(\mathrm{E})$ cells were added to target (T) cells (Pan02 or YAC-1) labeled with ${ }^{51} \mathrm{Cr}$ and co-cultured for $4 \mathrm{~h}$ at various E/T ratio. Percent cytotoxicity was calculated from the release of ${ }^{51} \mathrm{Cr}$ as follows: [(experimental release) - (target spontaneously release)]/ [(target maximum release) - (target spontaneously release)] x 100. Cytotoxicity against Pan02 cells showed the CTL activity and that against YAC-1 cells showed NK activity.

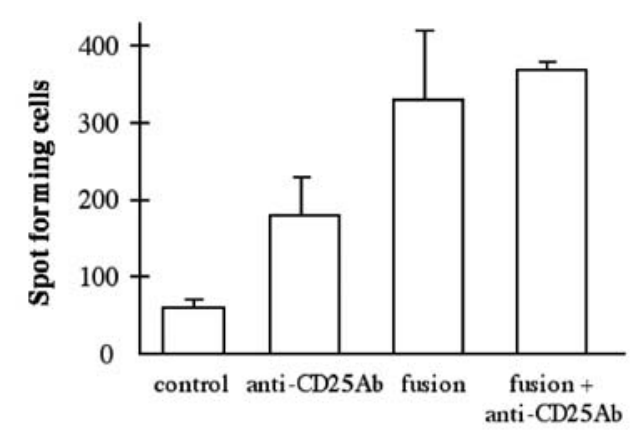

Figure 5. ELISPOT assay measured the secretion of IFN- $\gamma$ from splenocytes Twelve C57BL/6 mice were divided into four groups: PBS control, anti$\mathrm{CD} 25 \mathrm{Ab}$, fusion and fusion + anti-CD25Ab. Splenocytes isolated 7 days after tumor challenge subcutaneously were co-cultured with $1.0 \times$ Pan02 cells irradiated 30 Gy for a day. Splenocytes were collected and IFN- $\gamma$ secreting $\mathrm{T}$ cells were detected by using the ELISPOT assay. Figure represents means of spot forming cells with error bars.
NK cell activity than fusion group or anti-CD25Ab alone (61.3 vs. 48.6 and $38.1 \%$ at the $E / T$ ratio $50: 1, p=0.049$ and 0.03 , respectively). In addition, the NK cell activity for mice given fusion was significantly higher in comparison to that for mice given anti-CD25Ab $(\mathrm{p}<0.01)$, however no differences were observed in the NK cell activity between anti-CD25Ab monotherapy and the control group.

Assessment of cytokine secretion from effector cells. Twenty mice were divided into the previous four groups and sacrificed a week after tumor challenge. Briefly, splenocytes were collected and co-cultured with $1 \times 10^{5}$ Pan02 cells for $24 \mathrm{~h}$. Splenocytes and the culture supernatants were collected and analyzed using ELISPOT and a CBA Mouse Inflammation kit. In ELISPOT assays, each average number of SPC per $1 \times 10^{5}$ effector cells in the fusion + anti-CD25Ab, fusion, anti-CD25Ab and control was $379 \pm 10,314 \pm 86,197 \pm 38$ and $54 \pm 8$, respectively (Fig. 5). The combination of Treg depletion and fusion vaccination showed the highest ability for IFN- $\gamma$ production of effector cells. The spots for mice that received fusion was slightly lower than that for fusion + anti-CD25Ab, but was significantly higher in comparison to that for anti$\mathrm{CD} 25 \mathrm{Ab}(\mathrm{p}<0.01)$. In CBA assays, the mean levels of IFN- $\gamma$, IL-6 and IL-10 secretion are shown in Table I. There were no significant differences in other cytokines, such as TNF- $\alpha$, MCP-1 and IL-12p70. Combined therapy of Treg depletion and fusion vaccination showed the highest levels of IFN- $\gamma$ IL- 6 and IL-10 secretion of all groups. For mice given anti$\mathrm{CD} 25 \mathrm{Ab}$ monotherapy, secretion of IFN- $\gamma$ from effector cells was significantly lower than that for mice that received fusion alone or fusion + anti-CD25Ab therapy (17.9 vs. 210.9 or $594.9 \mathrm{pg} / \mathrm{ml}$, respectively; $\mathrm{p}<0.001)$. The ratio of IFN- $\gamma / \mathrm{IL}-6$ or IFN- $\gamma /$ IL-10 represents the relative ratio of Th1/Th2 cytokine production. The ratio of IFN- $\gamma /$ IL- 6 was significantly higher for the therapy including the fusion hybrids vaccine in comparison to that for the control $(\mathrm{p}<0.01)$. Meanwhile, for the anti-CD25Ab treatment regimens, the ratio of IFN- $\gamma / \mathrm{IL}-10$ was higher than that for fusion monotherapy $(\mathrm{p}<0.05)$, because IL-6 could be more dominant for anti-CD25Ab therapy in comparison to IL-10 for Th2 cytokines in the cytokine secretion profiles. In the Th17 assay, for the mice given the combination of fusion and anti-CD25Ab, significantly higher IL-17 secretion was demonstrated in effector cells after stimulation by Pan02 cells for $24 \mathrm{~h}$, in comparison to other 


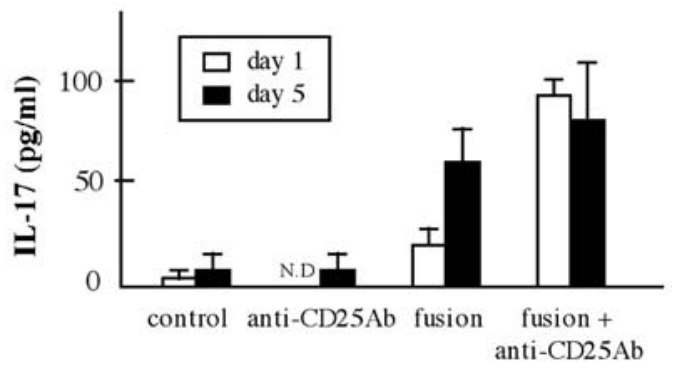

Figure 6. The assessment of IL-17 secretion from splenocytes by the enzymelinked immunosorbent assay (ELISA). Splenocytes were isolated from the mice subjected to a tumor challenge and they were co-cultured with Pan02 cells for 5 days. The supernatant of the culture was collected on days 1 or 5 and the secretion of IL-17 was measured. The secretion of IL-17 for either the 1-day or 5-day co-culture was not detected or 6.6 \pm 7.6 for anti-CD25Ab; $18.6 \pm 5.5$ or $58.3 \pm 21.7$ for fusion; $93.5 \pm 4.9$ or $79.5 \pm 32.5$ for fusion + antiCD25Ab, respectively. The IL-17 level in fusion + anti-CD25Ab were significantly higher than that in fusion alone for the 1-day co-culture $(\mathrm{p}<0.01)$. However, there were no significant differences between the fusion + anti-CD25Ab and fusion alone after the 5-day culture, although the IL-17 secretion for anti-CD25Ab alone was still low.

treatments (Fig. 6). However, for the mice given fusion only, IL-17 secretion increased to almost the same level as that for mice received with combined therapy after a 5-day stimulation. These results demonstrate that the combination of Treg depletion and fusion vaccination is the more effective in activation of both Th1 and Th17, in comparison to monotherapy with anti-CD25Ab or fusion hybrids.

\section{Discussion}

DC/TC fusion hybrid vaccines demonstrate a more effective anti-tumor immunity than either tumor lysate-pulsed DCs or mixtures of DCs and tumor cells in murine colon cancer models (8). Furthermore, DC/TC fusion hybrids can elicit CTLs specific to various tumor antigens and can be used for promising anti-tumor efficacy in future clinical trials because there is no need to identify tumor-associated antigens.

Tregs which are produced in the thymus are called natural Tregs and those which are altered from the naive $\mathrm{T}$ cells in the periphery are called induced Tregs. Natural Tregs are found in the regional lymph nodes where tissue-specific self antigens are presented by dendritic cells and they also migrate to infectious sites or tumors (24). Tregs maintain immune homeostasis by controlling peripheral tolerance against self-antigens. In recent studies, high levels of Tregs in the peripheral blood or tumor microenvironment have been revealed in patients with several kinds of cancer such as pancreatic, and ovarian cancer (25). High levels of Tregs could suppress the host spontaneous immune response against malignancies. In addition, it may also suppress specific anti-tumor immunity induced by adaptive immunotherapy or cancer vaccines. Several studies have shown that the depletion of Treg function and population may enhance the efficacy of immunotherapy in various cancer models (26).

In the present study, fusion + anti-CD25Ab combined treatment showed significantly improved survival in comparison to the fusion or anti-CD25Ab monotherapy groups. The anti-tumor efficacy of fusion hybrids or Treg depletion monotherapy could be essentially equivalent. However, the mechanism of immunotherapeutic efficacy could be different between fusion hybrids and antiCD25Ab therapy; because different types of effector cell activity and cytokine expression profiles were found in these experiments.

The CTL and NK cell activity for the fusion + anti$\mathrm{CD} 25 \mathrm{Ab}$ group was higher than that for the other treatment groups. These results suggested that Treg depletion and fusion vaccination induced effective anti-tumor immunity by induction of strong effector cells, especially NK cells. However, administration of anti-CD25Ab alone could not elicit NK cell activity in the present study. In assessing the cytokine profiles, both the ELISPOT and CBA assay demonstrated higher IFN- $\gamma$ levels for the fusion treatment groups, in comparison to anti-CD25Ab monotherapy. Wald et al reported that IFN- $\gamma$ stimulated the mobilization of NK cells into the circulation, but not their proliferation (27). Furthermore, only DC-activated NK cells are able to produce IFN- $\gamma$ and mediate anti-tumor protection (28). Therefore, DC-based therapy, such as DC/TC fusion hybrids are essential for induction of NK cells with anti-tumor potential in Treg depletion therapy.

Fusion hybrid vaccine elicits tumor-specific CTL activity, as well as NK cells in colon adenocarcinoma models (8). However, in the present study, fusion alone failed to induce tumor-specific CTL activity, although significant NK cell activity was found in mice given the fusion vaccine. The therapeutic efficacy for DC/TC vaccine is insufficient in murine renal cell carcinoma, $\mathrm{RenCa}$, which expresses a high level of TGF- $\beta$, although, enhanced therapeutic efficacy was observed when DC/TC fusion expressing a soluble TGF- $\beta$ receptor was used (29). Therefore, in Pan02 models which also produce TGF- $\beta$, Treg suppressed anti-tumor immunity induced by DC/TC fusion hybrids through secreted TGF- 3 . Prasad et al demonstrated that the depletion of Treg did not reveal the activity of CTLs specific for melanocyte differentiation antigens in B16-F10 mouse melanoma models (30). Significant differences in the CTL activity was found only between fusion + anti-CD25Ab combined therapy and control at $\mathrm{E} / \mathrm{T}$ ratio of $50: 1$. The results suggested that the Treg depletion by anti-CD25Ab could potentially enhance the CTL activity induced by the DC/TC fusion vaccine, however, an additional strategy is necessary to induce the cancer-specific CTL efficiently. Some activated CD4- or CD8-positive T cells, which expressed the CD25 antigen might have been eliminated by anti-CD25Ab in this experiment. In the present study, elimination of some activated $\mathrm{T}$ cells might have mitigated the induction of tumor-specific CTL and the anti-tumor effect by DC/TC fusion. Currently some cell-surface molecules that are expressed predominantly on natural Treg include, cytotoxic T lymphocyte associated antigen-4 (CTLA-4), folate receptor 4 (FR4), and glucocorticoid-induced tumor necrosis factor receptor family-related receptor (GITR) (31). Monoclonal Abs against these molecules might be exploited to suppress Treg function specifically. In the future, this problem could be resolved by a specific Ab to deplete Tregs without downregulation of anti-tumor activity for effector T cells. A specific $\mathrm{Ab}$ against Treg, tumor-specific CTL induction could be 
enhanced more effectively in combined immunotherapy with $\mathrm{DC} / \mathrm{TC}$ fusion hybrid vaccine.

In the current model, the Treg depletion alone resulted in anti-tumor efficacy in vivo similar to fusion monotherapy. However, no NK cell activity was seen and lower levels of Th1 cytokines, such as IFN- $\gamma$ was found in mice treated with anti-CD25Ab alone. Foxp3 inhibits the Th17 lineage, but the role of IL-17 in anti-tumor immunity is still unknown (32). A few studies have suggested that Th17 could assume a role in tumor prevention (33). Meanwhile, IL-6 induces the differentiation of Th17 cells (34). The addition of anti$\mathrm{CD} 25 \mathrm{Ab}$ up-regulated IL-6 secretion with a higher Th1/Th2 ratio in comparison to fusion alone. In our study, an early elevation of IL-17 secretion from effector cells was observed for the combination of fusion hybrids and anti$\mathrm{CD} 25 \mathrm{Ab}$. Therefore, it is theoretically possible that the anti-CD25Ab treatment might enhance the Th17 response and could contribute to improving tumor growth inhibition or survival in combination with DC/TC fusion hybrids. Future studies are therefore needed to examine the effect of fusion + Treg depletion strategies on the Th17 response in vivo, because the correlations among Th1, Th2 and Th17 in the anti-tumor immunity of the present combined therapy is still unknown.

In conclusion, the combined immunotherapy of a DC/TC fusion hybrid vaccine and anti-CD25Ab elicited the antitumor immune response of NK cells, supported by high levels of IFN- $\gamma$, Th1/Th2 ratio and Th17. Furthermore, the combined therapy of Treg depletion and DC/TC fusion vaccine could potentially enhance the CTL activity. In the future, the mechanism of anti-tumor activity by Treg depletion therapy should be investigated by an analysis of various cytokine expression profiles, especially Th17 as well as Th1/Th2.

\section{References}

1. Porgador A and Gilboa E: Bone marrow-generated dendritic cells pulsed with a class I-restricted peptide are potent inducers of cytotoxic T lymphocytes. J Exp Med 182: 255-260, 1995.

2. Steinman RM: The dendritic cell system and its role in immunogenicity. Annu Rev Immunol 9: 271-296, 1991.

3. Kim HS, Choo YS, Koo T, et al: Enhancement of antitumor immunity of dendritic cells pulsed with heat-treated tumor lysate in murine pancreatic cancer. Immunol Lett 103: 142-148, 2006.

4. Wolkers MC, Brouwenstijn N, Bakker AH, Toebes M and Schumacher TN: Antigen bias in T cell cross-priming. Science 304: 1314-1317, 2004

5. Galea-Lauri J, Wells JW, Darling D, Harrison P and Farzaneh F: Strategies for antigen choice and priming of dendritic cells influence the polarization and efficacy of antitumor T-cell responses in dendritic cell-based cancer vaccination. Cancer Immunol Immunother 53: 963-977, 2004.

6. Kao JY, Zhang M, Chen CM and Chen JJ: Superior efficacy of dendritic cell-tumor fusion vaccine compared with tumor lysatepulsed dendritic cell vaccine in colon cancer. Immunol Lett 101: 154-159, 2005.

7. McConnell EJ, Pathangey LB, Madsen CS, Gendler SJ and Mukherjee P: Dendritic cell-tumor cell fusion and staphylococcal enterotoxin B treatment in a pancreatic tumor model. J Surg Res 107: 196-202, 2002.

8. Yasuda T, Kamigaki T, Kawasaki K, et al: Superior anti-tumor protection and therapeutic efficacy of vaccination with allogeneic and semiallogeneic dendritic cell/tumor cell fusion hybrids for murine colon adenocarcinoma. Cancer Immunol Immunother 56: 1025-1036, 2007.
9. Galea-Lauri J, Darling D, Mufti G, Harrison P and Farzaneh F: Eliciting cytotoxic T lymphocytes against acute myeloid leukemia-derived antigens: evaluation of dendritic cell-leukemia cell hybrids and other antigen-loading strategies for dendritic cell-based vaccination. Cancer Immunol Immunother 51: 299-310, 2002.

10. Gong J, Avigan D, Chen D, et al: Activation of antitumor cytotoxic T lymphocytes by fusions of human dendritic cells and breast carcinoma cells. Proc Natl Acad Sci USA 97: 2715-2718, 2000.

11. Avigan D, Vasir B, Gong J, et al: Fusion cell vaccination of patients with metastatic breast and renal cancer induces immunological and clinical responses. Clin Cancer Res 10: 4699-4708, 2004.

12. Imura $\mathrm{K}$, Ueda $\mathrm{Y}$, Hayashi $\mathrm{T}$, et al: Induction of cytotoxic $\mathrm{T}$ lymphocytes against human cancer cell lines using dendritic cell-tumor cell hybrids generated by a newly developed electrofusion technique. Int J Oncol 29: 531-539, 2006.

13. Sakaguchi S, Sakaguchi N, Shimizu J, et al: Immunologic tolerance maintained by $\mathrm{CD} 25^{+} \mathrm{CD} 4^{+}$regulatory $\mathrm{T}$ cells: their common role in controlling autoimmunity, tumor immunity, and transplantation tolerance. Immunol Rev 182: 18-32, 2001.

14. Sakaguchi S: Naturally arising $\mathrm{CD}^{+}$regulatory $\mathrm{T}$ cells for immunologic self-tolerance and negative control of immune responses. Annu Rev Immunol 22: 531-562, 2004.

15. Fontenot JD and Rudensky AY: A well adapted regulatory contrivance: regulatory $\mathrm{T}$ cell development and the forkhead family transcription factor Foxp3. Nat Immunol 6: 331-337, 2005.

16. Malek TR and Bayer AL: Tolerance, not immunity, crucially depends on IL-2. Nat Rev Immunol 4: 665-674, 2004.

17. Read S, Malmstrom V and Powrie F: Cytotoxic T lymphocyteassociated antigen 4 plays an essential role in the function of CD25(+)CD4 $(+)$ regulatory cells that control intestinal inflammation. J Exp Med 192: 295-302, 2000.

18. Sakaguchi S, Yamaguchi T, Nomura T and Ono M: Regulatory T cells and immune tolerance. Cell 133: 775-787, 2008.

19. Ermann J, Szanya V, Ford GS, Paragas V, Fathman CG and Lejon K: CD4(+)CD25(+) T cells facilitate the induction of T cell anergy. J Immunol 167: 4271-4275, 2001.

20. Liyanage UK, Goedegebuure PS, Moore TT, et al: Increased prevalence of regulatory $\mathrm{T}$ cells (Treg) is induced by pancreas adenocarcinoma. J Immunother 29: 416-424, 2006.

21. Dannull J, Su Z, Rizzieri D, et al: Enhancement of vaccinemediated antitumor immunity in cancer patients after depletion of regulatory T cells. J Clin Invest 115: 3623-3633, 2005.

22. Lutz MB, Kukutsch N, Ogilvie AL, et al: An advanced culture method for generating large quantities of highly pure dendritic cells from mouse bone marrow. J Immunol Methods 223: 77-92, 1999.

23. Wang J, Saffold S, Cao X, Krauss J and Chen W: Eliciting T cell immunity against poorly immunogenic tumors by immunization with dendritic cell-tumor fusion vaccines. J Immunol 161: 5516-5524, 1998.

24. Samy ET, Parker LA, Sharp CP and Tung KS: Continuous control of autoimmune disease by antigen-dependent polyclonal $\mathrm{CD} 4{ }^{+} \mathrm{CD} 25^{+}$regulatory $\mathrm{T}$ cells in the regional lymph node. J Exp Med 202: 771-781, 2005.

25. Ikemoto $\mathrm{T}$, Yamaguchi $\mathrm{T}$, Morine $\mathrm{Y}$, et al: Clinical roles of increased populations of Foxp $3^{+} \mathrm{CD} 4^{+} \mathrm{T}$ cells in peripheral blood from advanced pancreatic cancer patients. Pancreas 33: 386-390, 2006.

26. Viehl CT, Moore TT, Liyanage UK, et al: Depletion of CD4 ${ }^{+}$ $\mathrm{CD} 25^{+}$regulatory $\mathrm{T}$ cells promotes a tumor-specific immune response in pancreas cancer-bearing mice. Ann Surg Oncol 13: 1252-1258, 2006.

27. Wald O, Weiss ID, Wald H, et al: IFN-gamma acts on T cells to induce NK cell mobilization and accumulation in target organs. J Immunol 176: 4716-4729, 2006.

28. Karimi K, Boudreau JE, Fraser K, et al: Enhanced antitumor immunity elicited by dendritic cell vaccines is a result of their ability to engage both CTL and IFN gamma-producing NK cells. Mol Ther 16: 411-418, 2008.

29. Zhang M, Berndt BE, Chen JJ and Kao JY: Expression of a soluble TGF-beta receptor by tumor cells enhances dendritic cell/tumor fusion vaccine efficacy. J Immunol 181: 3690-3697, 2008.

30. Prasad SJ, Farrand KJ, Matthews SA, Chang JH, McHugh RS and Ronchese F: Dendritic cells loaded with stressed tumor cells elicit long-lasting protective tumor immunity in mice depleted of $\mathrm{CD} 4{ }^{+} \mathrm{CD} 25^{+}$regulatory T cells. J Immunol 174: 90-98, 2005. 
31. Stephens GL, McHugh RS, Whitters MJ, et al: Engagement of glucocorticoid-induced TNFR family-related receptor on effector $\mathrm{T}$ cells by its ligand mediates resistance to suppression by CD4 ${ }^{+} \mathrm{CD} 25^{+} \mathrm{T}$ cells. J Immunol 173: 5008-5020, 2004.

32. Zhou L, Lopes JE, Chong MM, et al: TGF-beta-induced Foxp3 inhibits $\mathrm{T}(\mathrm{H}) 17$ cell differentiation by antagonizing ROR gammat function. Nature 453: 236-240, 2008.
33. Kryczek I, Wei S, Zou L, et al: Cutting edge: Th17 and regulatory $\mathrm{T}$ cell dynamics and the regulation by IL- 2 in the tumor microenvironment. J Immunol 178: 6730-6733, 2007.

34. Boe A, Baiocchi M, Carbonatto M, Papoian R and SerlupiCrescenzi O: Interleukin 6 knock-out mice are resistant to antigen-induced experimental arthritis. Cytokine 11: 1057-1064, 1999. 